\title{
EDUCAÇÃO NUTRICIONAL EM UNIVERSITÁRIOS E ESTRATÉGIAS PARA PROMOÇÃO DE SAÚDE INSTITUCIONAL: REVISÃO INTEGRATIVA
}

\author{
Nutrition education for university students and strategies for institutional health promotion: an \\ integrative review
}

\section{Educación nutricional de universitarios y estrategias de promoción de la salud institucional: revisión integrativa}

\author{
Marina Carvalho Berbigier \\ Universidade Federal de Ciências da Saúde de Porto Alegre - UFCSPA (RS) - Brasil \\ Cleidilene Ramos Magalhães \\ Universidade Federal de Ciências da Saúde de Porto Alegre - UFCSPA (RS) - Brasil
}

\section{RESUMO}

Objetivo: Refletir sobre o ambiente universitário como espaço promotor de saúde nutricional enfatizando as estratégias utilizadas. Métodos: Realizou-se uma revisão integrativa de literatura nas bases eletrônicas Scopus, MEDLINE, LILACS e SciELO utilizando os descritores: Nutrition Education, University Students, College Studens e Health Promotion. Incluiram-se os estudos publicados entre os anos de 2010 e 2015, originais, em língua portuguesa, inglesa ou espanhola, e que tivessem estratégia de intervenção nutricional em população de estudantes universitários bem definida. Dos 649 estudos revisados, após análise de títulos, resumos e texto integral, foram incluídos 18 artigos, todos realizados fora do Brasil. Resultados: Observou-se o ambiente universitário como potencial promotor de saúde no que tange ao contexto internacional, porém ainda incipiente no contexto nacional. Resultados positivos de mudança de comportamento alimentar após intervenções foram observados, embora sem avaliações a longo prazo. As principais abordagens identificadas foram intervenções práticas e teóricas com base na teoria social cognitiva e na motivacional. Conclusão: O ambiente universitário possui grande potencial para promoção de saúde nutricional e as estratégias mais efetivas foram as que apresentaram uma condução baseada na teoria social cognitiva. No entanto, esse ambiente tem sido pouco explorado em nível nacional, e, para mudar essa realidade, faz-se necessária uma ressignificação das estratégias utilizadas e das políticas públicas e ações intersetoriais que sustentem essa prática, inserindo-a na identidade social do ensino superior no Brasil.

Descritores: Educação Alimentar e Nutricional; Universidades; Educação; Promoção da Saúde.

\section{ABSTRACT}

Objective: To reflect on the university environment as a nutritional health promoting space with a focus on the strategies used. Methods: An integrative review of articles published in SCOPUS, MEDLINE (PubMed), LILACS and SciELO databases under the following descriptors: Nutrition Education, University Students, College Students, and Health Promotion. The review included original studies published between 2010 and 2015 in Portuguese, English or Spanish that reported a nutritional intervention strategy carried out with well-defined population of university students. Only 18 articles out of the 649 studies reviewed were included after the analysis of titles, abstracts and full text. All the studies were conducted outside Brazil. Results: The university environment was found to be a potential health promoting space in the international context that is still incipient in the national context. Positive changes in dietary behaviors were reported, although there were no long-term assessments. The main strategies identified were practical and theoretical interventions based on the social cognitive theory and motivation theory. Conclusion: The university environment has great potential for the promotion of nutritional health and the most effective strategies were those based on the social cognitive theory. However, this environment has been little explored in the national context. Changing this reality requires the redefinition of the strategies used and of the public policies and intersectoral actions that support such a practice so that it can be inserted in the social identity of higher education in Brazil.

Descriptors: Food and Nutrition Education; Universities; Education; Health Promotion. 


\section{RESUMEN}

Objetivo: Reflexionar sobre el ambiente de la universidad como espacio de promoción de salud nutricional con énfasis para las estrategias utilizadas. Métodos: Se realizó una revisión integrativa de la literatura en las bases de datos electrónicas Scopus, MEDLINE, LILACS y SciELO con los descriptores: Nutrition Education, University Students, College Studens y Health Promotion. Se incluyó los estudios publicados entre 2010 y 2015, originales, en el idioma portugués, inglés o español y que hubieran utilizado una estrategia de intervención nutricional en una población de estudiantes universitarios bien definida. Tras el análisis de los títulos, resúmenes y texto completo de los 649 estudios revisados, 18 artículos fueron incluidos y todos internacionales. Resultados: Se observó el ambiente de la universidad como un potencial promotor de la salud respecto el contexto internacional, aunque incipiente en el contexto nacional. Fueron observados resultados positivos de cambio de conducta alimentaria después de las intervenciones aunque sin evaluaciones a largo plazo. Los principales abordajes identificados fueron las intervenciones prácticas y teóricas basadas en la teoría social cognitiva y emocional. Conclusión: El ambiente de la universidad tiene gran potencial para la promoción de la salud nutricional y las estrategias más efectivas fueron las que presentaron una conducción basada en la teoría social cognitiva. Sin embargo, ese ambiente ha sido poco explorado a nivel nacional. Para cambiar esa realidad es necesario otra significación de las estrategias utilizadas y de las políticas públicas y acciones intersectoriales que sostengan esa práctica, incluyéndola en la identidad social de la educación superior de Brasil.

Descriptores: Educación Alimentaria y Nutricional; Universidades; Educación; Promoción de la Salud.

\section{INTRODUÇÃO}

As condutas de saúde estabelecidas no decorrer da vida têm impacto importante no processo de saúde e doença dos indivíduos. A Organização Mundial da Saúde (OMS) define saúde como uma interação complexa entre bem-estar físico, mental, espiritual e social. Nesse contexto, são englobados diversos fatores já consolidados, dentre os quais o comportamento alimentar se destaca como um determinante fator modificável ${ }^{(1)}$.

O cenário atual apresenta mudanças de conduta de saúde e hábitos em adultos jovens, faixa etária onde se encontram a maior parte dos estudantes universitários, com uma importante tendência a um estilo de vida menos saudável, ratificado por um panorama significativo de estresse, sedentarismo e alimentação inadequada ${ }^{(2-9)}$. É sabido que a transição epidemiológica no Brasil, associada à transição nutricional, proporcionou um aumento expressivo de doenças crônicas não transmissíveis e obesidade, consideradas um importante problema de saúde pública, acarretando em aumento de risco cardiovascular, piora de qualidade de vida e desenvolvimento precoce de câncer ${ }^{(10,11)}$. Embora esse fenômeno seja observado em todas as faixas etárias, os indicadores elevados em jovens geram maior preocupação, no que tange ao impacto relacionado à morbi-mortalidade no Brasil e no mundo ${ }^{(1,12,13)}$.

Os espaços coletivos, caso das instituições de ensino superior, podem ser importantes promotores de saúde, com a adequação da alimentação como um dos aspectos fundamentais, sendo incentivadas por políticas e diretrizes, como as propostas através da Política Nacional de Alimentação e Nutrição (PNAN) ${ }^{(14)}$ e corroboradas por programas como o Programa Nacional de Alimentação do Escolar (PNAE) ${ }^{(15,16)}$, que há anos e em constante evolução contribui de maneira importante para o desenvolvimento de crianças e adolescentes em idade escolar. Ainda que a educação superior não seja parte do PNAE, as Escolas Promotoras de Saúde (EPS) ${ }^{(17-19)}$ assumem a responsabilidade por esses espaços com o objetivo de promover saúde em qualquer ambiente coletivo de ensino, formal ou não, fomentando estratégias de educação nutricional e promoção de saúde que acarretem em problematização e modificação desses ambientes em prol de melhorias para comunidade acadêmica usuária, construindo, dessa forma, um núcleo de promoção de saúde local coletivo.

Porém, no contexto brasileiro, a maior parte dos estudos que abrangem as estratégias de modificação de comportamentos alimentares em ambiente de ensino retrata as fases de educação básica ${ }^{(20-23)}$, muito justificado pelo fato da educação superior não fazer parte do PNAE.

No entanto, uma vez que a PNAN incentiva a promoção de educação nutricional em todos os espaços coletivos, isso incluiria os universitários. Além disso, contextualizada a situação emergente de agravos à saúde nesse grupo, no que se refere a hábitos alimentares, faz-se necessário conhecer as estratégias de educação nutricional que já são utilizadas com alunos de graduação e sua efetividade em estabelecer esse ambiente como espaço promotor de saúde nutricional. Portanto, esse estudo objetivou refletir sobre o ambiente universitário como espaço promotor de saúde nutricional enfatizando as estratégias utilizadas.

\section{MÉTODOS}

Esse estudo consiste em uma revisão integrativa da literatura, método que possibilita análise e síntese de resultados de maneira sistematizada. A elaboração dessa revisão respeitou todas as fases pré-estabelecidas para a sua realização, abrangendo as seguintes etapas: formulação das questões norteadoras; seleção e obtenção dos artigos conforme critérios de inclusão e 
exclusão; coleta de dados; avaliação dos estudos selecionados; discussão e interpretação dos resultados, e apresentação da revisão ${ }^{(24)}$.

A principal pergunta norteadora para a revisão foi: $\mathrm{O}$ ambiente universitário é um espaço promotor de saúde nutricional? Ressalta-se que os objetivos da revisão sugeriram a necessidade de demais perguntas adjacentes à principal, sendo essas então definidas por: Quais estratégias de educação nutricional são utilizadas em ambiente universitário? Quais profissionais são responsáveis por estratégias de promoção de saúde nutricional em ambiente universitário? Quais os resultados das estratégias de educação nutricional em ambiente universitário?

A pesquisa foi realizada nos meses de outubro e novembro de 2015 e foram incluídos os estudos publicados, na íntegra, entre os anos de 2010 e 2015, em língua portuguesa, inglesa ou espanhola, e somente artigos originais revisados por pares e indexados nas bases de dados eletrônicas pesquisadas, ou seja, U. S. National Library of Medicine (MEDLINE), Scopus, Scientific Eletronic Library Online (SciELO) e Literatura Latino Americana e do Caribe em Ciências da Saúde (LILACS). Foram utilizadas as seguintes palavras-chave: Nutrition Eduacation, University Students, College Studens e Health Promotion, através das seguintes equações de busca: Nutrition education AND (University Students OR college students); Nutrition Education AND University Students e Nutrition Education AND Health Promotion AND University Students.

Para a inserção dos estudos na revisão integrativa, deveriam descrever a estratégia de educação nutricional utilizada e a população deveria incluir estudantes universitários saudáveis, com diferentes diagnósticos de estado nutricional. As avaliações das estratégias poderiam ser qualitativas e/ou quantitativas, através de análise estatística. Os trabalhos que não contemplaram esse formato foram excluídos. O processo de seleção dos artigos para análise na íntegra está descrito na Figura 1.

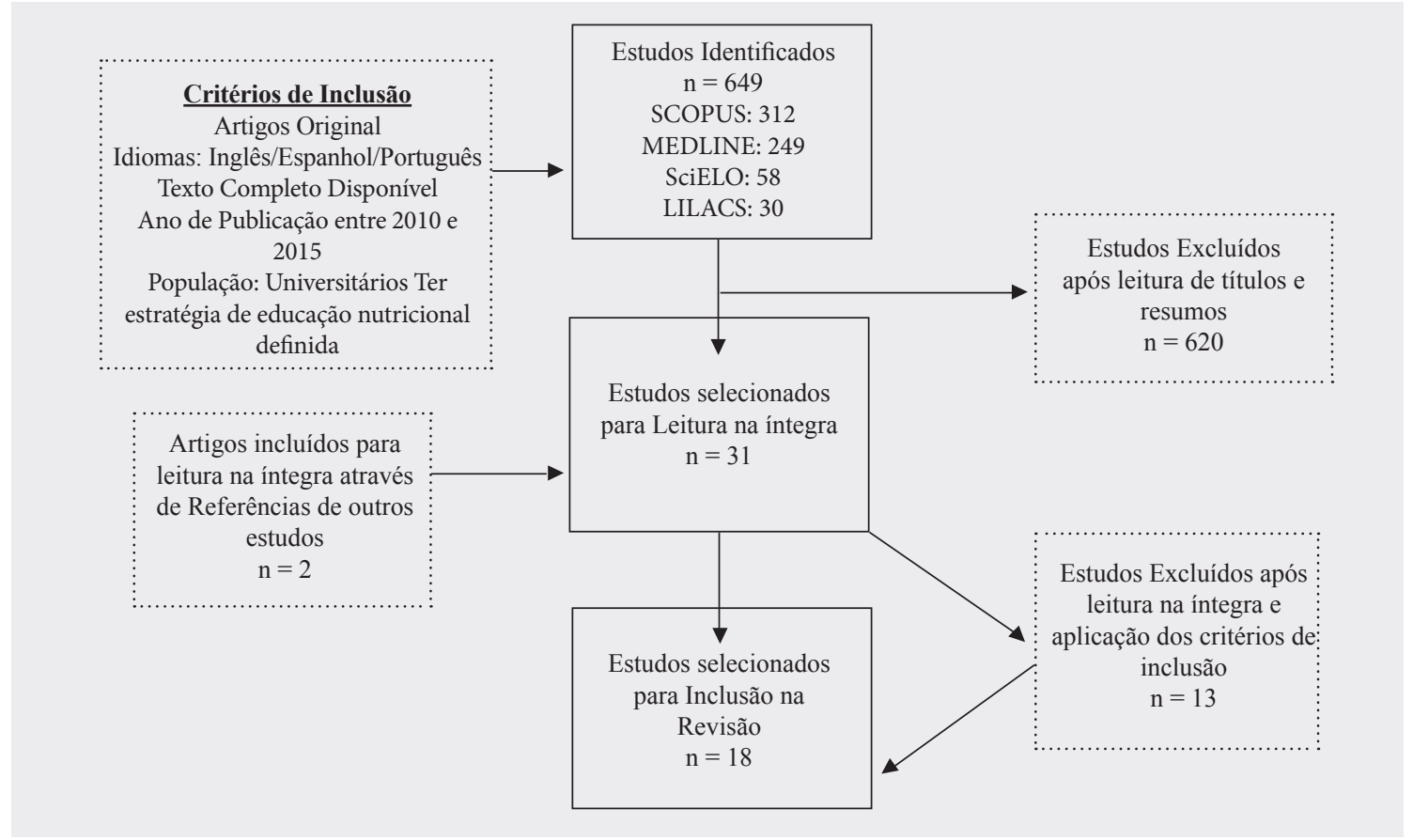

Figura 1 - Fluxograma dos artigos incluídos no estudo.

\section{RESULTADOS}

Dentre os estudos selecionados $(\mathrm{n}=18)$ que atenderam aos critérios de inclusão, a maior parte aconteceu nos Estados Unidos (7 estudos), seguido pelo Canadá (3 estudos), totalizando 55,5\% da amostra. No entanto, observou-se distribuição em 9 diferentes países (Estados Unidos da América - EUA, Canadá, Argentina, Colômbia, Índia, Malásia, Nigéria, Alemanha e Espanha), abrangendo 5 continentes (América do Sul, América do Norte, Europa, África e Ásia). Todos os estudos foram realizados dentro de instituições de ensino superior, sendo 3 publicações em língua espanhola e 15 em língua inglesa. A análise dos artigos evidencia que o ambiente universitário é um importante espaço promotor de saúde, porém ainda incipiente em estratégias no Brasil, uma vez que não foi possível incluir nenhum estudo desenvolvido em âmbito nacional, sobretudo por ausência de estratégias de intervenção bem descritas.

Os estudos apresentaram uma ou mais estratégias de intervenção com o objetivo de educação nutricional, doze (66,6\%) deles realizaram estratégia única e/ou principal; e seis $(33,3 \%)$ estratégias mistas. Para análise das estratégias encontradas, foram divididas em 6 abordagens diferentes, apresentadas e distribuídas da seguinte forma: 1) Oficinas/palestras/jornadas/ exposições/encontros com a utilização de material informativo impresso e ou ilustrativo (61,1\% dos estudos, $n=11)$; 2$)$ Cursos 
presenciais, de longa duração, com período superior a 8 encontros $(22,2 \%$ dos estudos, $n=4)$; 3$)$ Cursos ou programas através da internet $(33,3 \%$ dos estudos, $n=6)$; 4) Programas de incentivo através de mensagens por telefone (11,1\% dos estudos, $n=2)$; 5) Rotulagem de alimentos vendidos no ambiente universitário com sinalização de risco (11,1\% dos estudos , n=2); e 6$)$ Aulas práticas de culinária $(5,5 \%$ dos estudos, $n=1)$.

Em relação às diferentes abordagens realizadas durante as estratégias, observou-se, principalmente, um apelo motivacional, abordagens baseadas na Teoria Social Cognitiva, com enfoque na autoeficácia e autorregulação em saúde, e outras apenas com base em transmissão de conteúdo, em que só a informação propriamente dita foi fornecida ao participante.

A maior parte dos estudos apresentou abordagens mistas em suas estratégias, ou seja, utilizou mais de uma estratégia de intervenção em seus programas. Os principais atores identificados como proponentes dessas estratégias foram nutricionistas e profissionais de equipes da área da saúde atuantes na universidade, estudantes dos cursos de graduação e pós-graduação nas mesmas áreas e profissionais de apoio das instituições.

Em relação aos resultados obtidos, atenção especial foi dada à discussão relacionada à rede de acontecimentos que sustentam hábitos inadequados de alimentação por parte da população estudada, viabilizando intervenções oportunas para as principais necessidades dos participantes. Os principais motivos destacados como justificativa para o estilo de vida que coloca esses jovens em risco para o desenvolvimento de doenças metabólicas foram: excesso de demanda acadêmica, (resultando em pouco tempo para prática de atividade física e produção de uma alimentação adequada), aumento de responsabilidades pessoais (muitos alunos deixam a casa dos pais no momento em que ingressam na universidade), falta de opções saudáveis disponíveis nas universidades, falta de conhecimento a respeito de nutrição para realização de melhores escolhas, inaptidão para cozinhar, limitantes financeiros, estilo de vida desregrado (aumento do consumo de álcool e outras drogas e redução de horas de sono), constrangimento em fazer escolhas saudáveis e ser considerado exceção dentro do grupo de convívio.

Embora os resultados das intervenções ratifiquem o ambiente universitário com muito potencial para o desenvolvimento dessas ações, apresentando em sua totalidade resultados positivos de mudança de hábitos alimentares, perfil antropométrico e estilo de vida dos participantes após intervenção, apenas um estudo apresentou avaliação das estratégias em longo prazo, o que indica que ainda existe a necessidade de mais avaliações para corroborar com essas ações em definitivo.

A apresentação dos objetivos, estratégias e principais resultados dos estudos incluídos na revisão se apresentam descritas no quadro I.

Quadro I - Apresentação dos principais resultados dos estudos incluídos na revisão integrativa.

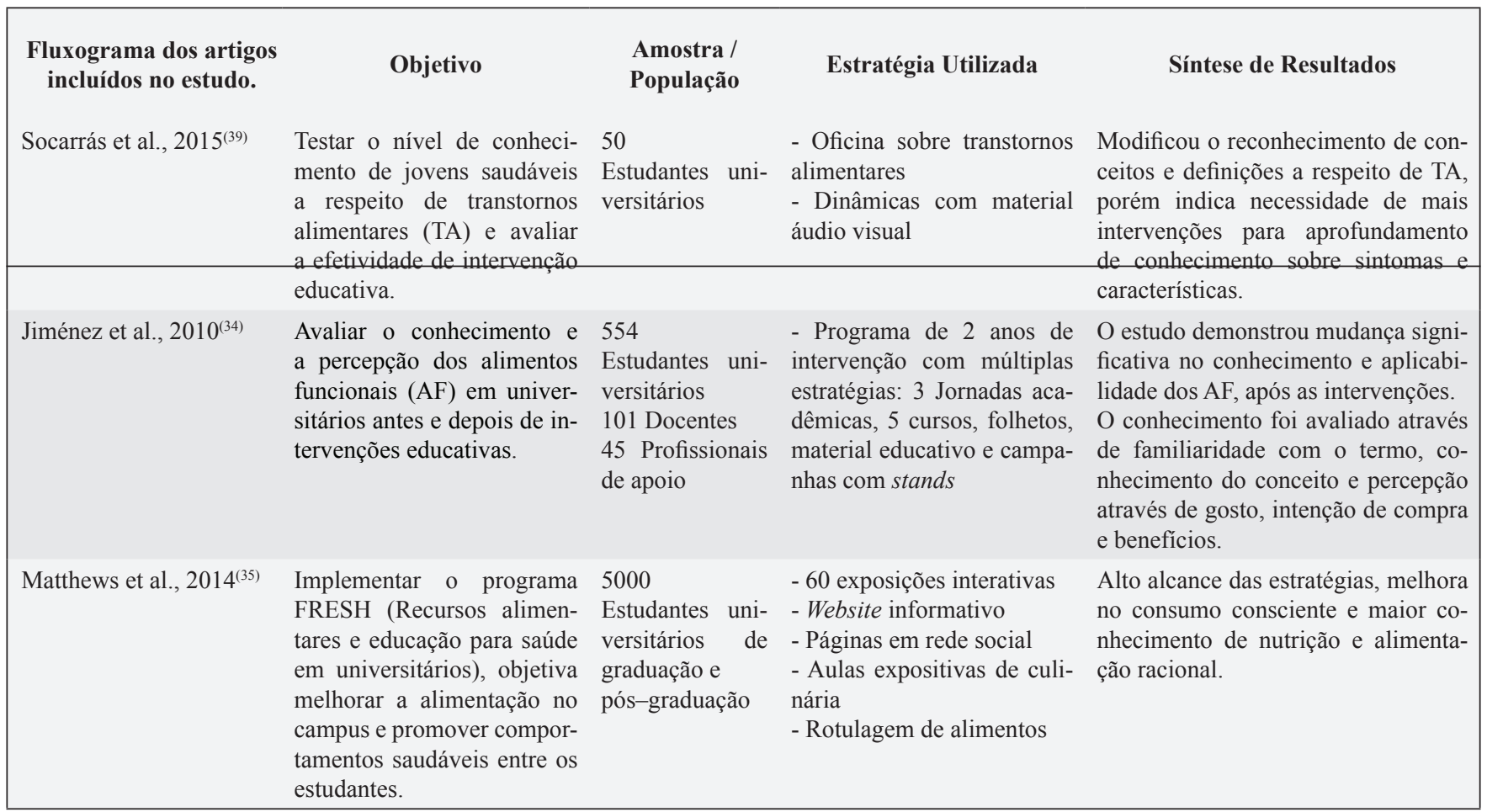




\begin{tabular}{|c|c|c|c|c|}
\hline $\begin{array}{l}\text { Becerra-Bulla et al., } \\
2011^{(40)}\end{array}$ & $\begin{array}{l}\text { Implementação do programa } \\
\text { Universidade Promotora de } \\
\text { Saúde. }\end{array}$ & $\begin{array}{l}\text { Não informado. } \\
\text { Estudantes uni- } \\
\text { versitários e co- } \\
\text { munidade aca- } \\
\text { dêmica }\end{array}$ & $\begin{array}{l}\text { - Estratégias educacionais } \\
\text { expositivas para consumo } \\
\text { de alimentação saudável } \\
\text { - Orientação nutricional in- } \\
\text { dividualizada }\end{array}$ & $\begin{array}{l}\text { Expansão do projeto para Docência, } \\
\text { pesquisa e extensão. } \\
\text { Docência: Melhorar a formação de } \\
\text { educadores nutricionais com refor- } \\
\text { mulação curricular dos cursos. } \\
\text { Pesquisa: Monitorar e avaliar conti- } \\
\text { nuamente o programa na universida- } \\
\text { de. } \\
\text { Extensão: Criação do projeto Am- } \\
\text { bientes saudáveis }\end{array}$ \\
\hline Lhakhang et al., 2014(36) & $\begin{array}{l}\text { Comparar duas sequências } \\
\text { de estratégias para aumentar } \\
\text { o consumo de frutas e vege- } \\
\text { tais em universitários. }\end{array}$ & $\begin{array}{l}224 \\
\text { Estudantes uni- } \\
\text { versitários resi- } \\
\text { dentes no cam- } \\
\text { pus }\end{array}$ & $\begin{array}{l}\text { - Intervenção motivacional } \\
\text { seguida de intervenção para } \\
\text { autorregulação } \\
\text { - Intervenção para autorre- } \\
\text { gulação seguida de interven- } \\
\text { ção motivacional }\end{array}$ & $\begin{array}{l}\text { - A intervenção que iniciou com es- } \\
\text { tratégias de autorregulação foi mais } \\
\text { efetiva para mudança de comporta- } \\
\text { mento alimentar quando comparada } \\
\text { com a intervenção que iniciou com } \\
\text { estratégia motivacional. }\end{array}$ \\
\hline Shahril et al., 2013 ${ }^{(32)}$ & $\begin{array}{l}\text { Avaliar intervenção com } \\
\text { método multimodal para } \\
\text { melhorar a qualidade do } \\
\text { consumo alimentar na uni- } \\
\text { versidade. }\end{array}$ & $\begin{array}{l}417 \\
\text { Estudantes uni- } \\
\text { versitários }\end{array}$ & $\begin{array}{l}\text { Multimodal } \\
\text { - Palestras } \\
\text { - Panfletos } \\
\text { - Mensagens de texto moti- } \\
\text { vacionais via celular }\end{array}$ & $\begin{array}{l}\text { Melhora significativa de hábitos ali- } \\
\text { mentares e consumo de macro e mi- } \\
\text { cronutrientes específicos. }\end{array}$ \\
\hline Feldman et al., $2013^{(37)}$ & $\begin{array}{l}\text { Avaliar e projetar uma forma } \\
\text { de promover seleção saudá- } \\
\text { vel de alimentos em pontos } \\
\text { de venda nas universidades. }\end{array}$ & $\begin{array}{l}39 \\
\text { Estudantes uni- } \\
\text { versitários }\end{array}$ & $\begin{array}{l}\text { - Grupos focais } \\
\text { - Material interativo com } \\
\text { diferentes perspectivas se- } \\
\text { miológicas de rotulagem } \\
\text { nutricional }\end{array}$ & $\begin{array}{l}\text { Os menus de codificação para ali- } \\
\text { mentos oferecidos na universidade } \\
\text { auxiliaram os alunos a fazerem me- } \\
\text { lhores escolhas alimentares. }\end{array}$ \\
\hline O'Donnell et al., 2014(44) & $\begin{array}{l}\text { Explorar a relação entre o } \\
\text { efeito do ajuste no objetivo } \\
\text { de consumo de frutas e ve- } \\
\text { getais e atividade física e o } \\
\text { próprio consumo durante } \\
\text { programa educativo via in- } \\
\text { ternet. }\end{array}$ & $\begin{array}{l}665 \\
\text { Estudantes uni- } \\
\text { versitários }\end{array}$ & $\begin{array}{l}\text { - } 10 \text { Intervenções de ensino } \\
\text { sobre alimentação saudável } \\
\text { e atividade física através da } \\
\text { WEB } \\
\text { - Determinação dos objeti- } \\
\text { vos e consumo semanal rea- } \\
\text { lizada pelos próprios alunos }\end{array}$ & $\begin{array}{l}\text { - Crescente aumento nos objetivos de } \\
\text { consumo de frutas e vegetais e o con- } \\
\text { sumo propriamente dito no decorrer } \\
\text { das semanas. Decidir sua meta indi- } \\
\text { vidual auxiliou que o objetivo fosse } \\
\text { alcançado pelos participantes. }\end{array}$ \\
\hline Dour et al., $2013^{(45)}$ & $\begin{array}{l}\text { Determinar o impacto glo- } \\
\text { bal do programa Webhealth } \\
\text { após } 3 \text { meses do término e } \\
\text { avaliar os componentes mo- } \\
\text { tivacionais das intervenções } \\
\text { em relação as mudanças de } \\
\text { comportamento e peso cor- } \\
\text { poral dos participantes. }\end{array}$ & $\begin{array}{l}653 \\
\text { Estudantes uni- } \\
\text { versitários }\end{array}$ & $\begin{array}{l}\text { - Educação Nutricional atra- } \\
\text { vés da WEB }\end{array}$ & $\begin{array}{l}\text { - Homens mantiveram maior consu- } \\
\text { mo de frutas e vegetais, porém tam- } \\
\text { bém maior IMC. Os participantes } \\
\text { que mantiveram escolhas alimentares } \\
\text { mais saudáveis foram aqueles que se } \\
\text { achavam mais motivados para o de- } \\
\text { senvolvimento do curso. }\end{array}$ \\
\hline $\begin{array}{l}\text { Harvey-Berino et al., } \\
2012^{(46)}\end{array}$ & $\begin{array}{l}\text { Explorar a viabilidade de } \\
\text { um programa online para } \\
\text { manejo de comportamento } \\
\text { de peso para estudantes uni- } \\
\text { versitários. }\end{array}$ & $\begin{array}{l}336 \\
\text { Estudantes uni- } \\
\text { versitários }\end{array}$ & $\begin{array}{l}\text { - Curso de educação nutri- } \\
\text { cional via internet } \\
\text { - Encontros em ambiente } \\
\text { virtual com nutricionista e } \\
\text { psicólogo } \\
\text { - Material educativo com- } \\
\text { plementar } \\
\text { - Dieta e orientações indivi- } \\
\text { dualizadas } \\
\text { - Receitas cardápio } \\
\text { - Diário alimentar }\end{array}$ & $\begin{array}{l}\text { Todas as alterações de peso foram } \\
\text { significativas de acordo com a meta. } \\
\text { Um curso online para suporte nutri- } \\
\text { cional e controle de peso é efetivo e } \\
\text { viável para essa população. }\end{array}$ \\
\hline Poddar et al., 2012(47) & $\begin{array}{l}\text { Modificar comportamentos, } \\
\text { através da teoria social cog- } \\
\text { nitiva, relacionados ao con- } \\
\text { sumo de produtos lácteos em } \\
\text { universitários }\end{array}$ & $\begin{array}{l}211 \\
\text { Estudantes uni- } \\
\text { versitários da } \\
\text { disciplina Saúde } \\
\text { Pessoal e Educa- } \\
\text { ção em Drogas }\end{array}$ & $\begin{array}{l}\text { - } 8 \text { semanas de cursos online } \\
\text { - eventos } \\
\text { Temática: } \\
\text { - Teoria Social Cognitiva } \\
\text { - Consumo de produtos ác- } \\
\text { teos }\end{array}$ & $\begin{array}{l}\text { Os estudantes aumentaram a autor- } \\
\text { regulação e o consumo de produtos } \\
\text { lácteos }\end{array}$ \\
\hline
\end{tabular}




\begin{tabular}{|c|c|c|c|c|}
\hline Pearson et al., $2013^{(48)}$ & $\begin{array}{l}\text { Comparar duas estratégias } \\
\text { de controle de obesidade } \\
\text { através de intervenções por } \\
\text { telefone: } \\
\text { 1) estratégia motivacional } \\
\text { 2) método APRENDER }\end{array}$ & $\begin{array}{l}78 \\
\text { Estudantes uni- } \\
\text { versitários com } \\
\text { IMC } \quad 30 \mathrm{~kg} / \mathrm{m}^{2} \\
\text { ou superior, sem } \\
\text { comorbidades }\end{array}$ & $\begin{array}{l}\text { - Intervenção via ligação te- } \\
\text { lefônica com temática sobre } \\
\text { estilo de vida. } \\
\text { 1) Grupo motivacional: con- } \\
\text { versa aberta } \\
\text { 2) Grupo APRENDER: con- } \\
\text { teúdo com teor } \\
\text { pré-determinado }\end{array}$ & $\begin{array}{l}\text { Os participantes do grupo APREN- } \\
\text { DER perderam mais peso, porém os } \\
\text { participantes do grupo motivacional } \\
\text { consumiram menos calorias. } \\
\text { Sobre a experiência, o grupo motiva- } \\
\text { cional relatou a abertura para reflexão } \\
\text { ótima para estabelecer suas metas, } \\
\text { mas sentiu falta de instruções especí- } \\
\text { ficas e o grupo APRENDER salien- } \\
\text { tou a importância das lições especi- } \\
\text { ficas mas sentiu falta de flexibilidade } \\
\text { e individualização de ações. Ambas } \\
\text { estratégias geraram resultados posi- } \\
\text { tivos. }\end{array}$ \\
\hline $\begin{array}{l}\text { Eun-Jeong Ha et al., } \\
2011^{(50)}\end{array}$ & $\begin{array}{l}\text { Estimar o consumo de grãos } \\
\text { integrais, suas fontes e de- } \\
\text { terminar se após realização } \\
\text { de curso sobre nutrição o } \\
\text { consumo aumenta, em estu- } \\
\text { dantes universitários. }\end{array}$ & $\begin{array}{l}90 \\
\text { Estudantes uni- } \\
\text { versitários }\end{array}$ & $\begin{array}{l}\text { - Disciplina Introdutória } \\
\text { sobre alimentação saudável, } \\
\text { atividade física e consumo } \\
\text { de grãos integrais com base } \\
\text { na teoria social cognitiva }\end{array}$ & $\begin{array}{l}\text { O curso se estabeleceu como uma } \\
\text { estratégia efetiva para o aumento do } \\
\text { consumo de grãos integrais em uni- } \\
\text { versitários. }\end{array}$ \\
\hline Anetor et al., 2012(41) & $\begin{array}{l}\text { Examinar o efeito da edu- } \\
\text { cação nutricional sobre os } \\
\text { hábitos alimentares de es- } \\
\text { tudantes de graduação para } \\
\text { prevenção de câncer de es- } \\
\text { tomago. }\end{array}$ & $\begin{array}{l}436 \\
\text { Estudantes uni- } \\
\text { versitários }\end{array}$ & $\begin{array}{l}\text { - } 8 \text { semanas de aula exposi- } \\
\text { tiva sobre alimentação sau- } \\
\text { dável e risco para câncer de } \\
\text { estomago }\end{array}$ & $\begin{array}{l}\text { Diferença significativa foi observada } \\
\text { nos hábitos alimentares protetivos } \\
\text { nos participantes que receberam a } \\
\text { intervenção. }\end{array}$ \\
\hline Higgins et al., 2010 $0^{(42)}$ & $\begin{array}{l}\text { Descrever práticas de bem } \\
\text { estar em estudantes univer- } \\
\text { sitários, desenvolver um } \\
\text { curso de educação em boas } \\
\text { práticas de saúde e avaliar } \\
\text { o impacto do curso sobre a } \\
\text { prática e o aprendizado dos } \\
\text { alunos. }\end{array}$ & $\begin{array}{l}1855 \\
\text { Estudantes uni- } \\
\text { versitários }\end{array}$ & $\begin{array}{l}\text { - Curso de educação em } \\
\text { saúde }\end{array}$ & $\begin{array}{l}\text { As análises dos resultados do curso } \\
\text { evidenciam melhora nos níveis de } \\
\text { consciência para realizar melhores } \\
\text { escolhas por parte dos participantes. } \\
\text { Incluindo alimentação e demais indi- } \\
\text { cadores de bem-estar e saúde. }\end{array}$ \\
\hline $\begin{array}{l}\text { Vijayapushpam et al., } \\
2010^{(43)}\end{array}$ & $\begin{array}{l}\text { Avaliar o impacto de uma } \\
\text { intervenção de educação em } \\
\text { saúde em sala de aula em } \\
\text { universitários de } 7 \text { institui- } \\
\text { ções na Índia. }\end{array}$ & $\begin{array}{l}687 \\
\text { Estudantes uni- } \\
\text { versitários }\end{array}$ & $\begin{array}{l}\text { - Palestras sobre saúde e ali- } \\
\text { mentação }\end{array}$ & $\begin{array}{l}\text { Melhora significativa na compreen- } \\
\text { são de educação e saúde. }\end{array}$ \\
\hline Ungar et al., 2013 & $\begin{array}{l}\text { Identificar qual intervenção } \\
\text { é mais efetiva para aumen- } \\
\text { to de consumo de frutas e } \\
\text { vegetais em universitários } \\
\text { o programa " } 5 \text { ao dia" ou o } \\
\text { "apenas mais um". }\end{array}$ & $\begin{array}{l}135 \\
\text { Estudantes uni- } \\
\text { versitários }\end{array}$ & $\begin{array}{l}\text { - Programa motivacional } \\
\text { com base na estratégia "5 ao } \\
\text { dia" e "apenas mais um" }\end{array}$ & $\begin{array}{l}\text { O programa motivacional " } 5 \text { ao dia" } \\
\text { obteve melhores resultados relacio- } \\
\text { nados ao aumento de consumo de } \\
\text { frutas e vegetais. }\end{array}$ \\
\hline Poddar et al., $2010^{(49)}$ & $\begin{array}{l}\text { Melhorar expectativas e } \\
\text { resultados de autoeficácia } \\
\text { e autorregulação através } \\
\text { da Teoria Social Cognitiva } \\
\text { (TSC) para o comportamen- } \\
\text { to e consumo de lácteos em } \\
\text { estudantes universitários. }\end{array}$ & $\begin{array}{l}294 \\
\text { Estudantes uni- } \\
\text { versitários ma- } \\
\text { triculados na } \\
\text { disciplina de } \\
\text { saúde pessoal }\end{array}$ & $\begin{array}{l}\text { - Curso de } 5 \text { semanas atra- } \\
\text { vés da WEB com a temática } \\
\text { Nutrição e Teoria Social } \\
\text { Cognitiva }\end{array}$ & $\begin{array}{l}\text { Os participantes que receberam a in- } \\
\text { tervenção tiveram aumento na autor- } \\
\text { regulação para o consumo de lácteos, } \\
\text { porém não no consumo efetivo, o que } \\
\text { indica que a TSC deve ser trabalhada } \\
\text { de maneira mais aprofundada. }\end{array}$ \\
\hline
\end{tabular}

\section{DISCUSSÃO}

Observando os estudos incluídos na presente revisão, é possível notar que todos foram realizados fora do Brasil, o que mostra que estratégias de educação nutricional organizadas, destinadas a universitários, são ainda incipientes.

No contexto brasileiro, mesmo que existam muitas estratégias bem estabelecidas para alunos de educação básica ${ }^{(20,21,25)}$, reforçadas pela PNAE, que se caracteriza como política pública de segurança nutricional de maior longevidade no país, e pela PNAN, que ratifica a necessidade de realização de promoção de saúde alimentar em todos os espaços coletivos de ensino ${ }^{(14,26)}$, observa-se que essa realidade não alcançou efetivamente o ensino superior. 
Em um estudo multicêntrico com 1.689 participantes com média de idade de 19 anos, realizado em universidades americanas, foram identificados índices de massa corporal (IMC) e circunferência da cintura (CC) elevados como indicadores de alto risco em saúde, bem como, excesso de peso em mais de $20 \%$ da população ${ }^{(5)}$. Estudos semelhantes realizados na Grécia (com 390 estudantes de medicina), no Reino Unido (com 524 estudantes de 37 universidades diferentes), no Chile (com 799 estudantes de 4 universidades) e na França (com 3457 estudantes de 8 universidades), observaram índices igualmente preocupantes, tais como: $30 \%$ da população masculina com excesso de peso e baixo conhecimento nutricional; $24 \%$ de excesso de peso global e dieta pobre em nutrientes; baixa média de adesão à dieta mediterrânea e frequência de atividade física semanal (inferior a duas vezes); e alta prevalência de transtornos alimentares associados a demais riscos comportamentais, como estresse e depressão respectivamente ${ }^{(6-8,27)}$.

No Brasil, os índices não se apresentam diferentes. Em um estudo realizado com 1.503 estudantes universitários no nordeste, foram observados baixos índices de atividade física ${ }^{(28)}$; assim como, em outro trabalho semelhante realizado na mesma região, foi evidenciado altos fatores de risco cardiovasculares, como tabagismo, sedentarismo, excesso de peso, inadequação de consumo calórico e diabetes em alunos da mesmo faixa etária ${ }^{(3)}$.

Esses achados em adultos jovens entre 18 e 24 anos corroboram com a epidemia de doenças cardiovasculares ${ }^{(29)}$ e metabólicas $^{(30,31)}$ observadas atualmente. Ressalta-se que, ao lançar o Plano de Ações Estratégicas para o Enfrentamento de Doenças Crônicas não Transmissíveis 2011-2022, o Brasil assume o compromisso de prevenção e promoção de saúde que contemple todas as idades e espaços, no que se refere aos fatores de risco modificáveis (tabagismo, álcool, alimentação, inatividade e obesidade) relacionados à predisposição para essas doenças ${ }^{(32)}$. A soma desses fatores faz com que estudos que se proponham a desenvolver essa área sejam emergentes.

Muitos motivos já foram explorados para justificar a mudança negativa de hábitos alimentares em estudantes universitários. Ressaltam-se questões financeiras relacionadas com a saída do jovem da casa da família para estudar, estresse com as atividades acadêmicas, ausência de tempo para uma maior dedicação à produção da sua própria alimentação e/ou atenção a horários, falta de habilidade para cozinhar e de conhecimento de nutrição para realização de escolhas mais saudáveis, conforme apresentado anteriormente $^{(33-35)}$

Através dos estudos selecionados para o presente artigo, as estratégias que melhor apresentaram resultados para mudança de comportamento nessa população e seus principais limitantes foram observadas.

Estudos realizados na América do Norte constataram que a identificação dos alimentos oferecidos no campus das universidades, referente à sua composição nutricional, foi capaz de modificar as escolhas alimentares realizadas pelos alunos durante as refeições. Todavia, ressaltaram que somente a informação das características nutricionais de determinados alimentos não se apresentou como uma ação efetiva, pois a ausência de conhecimento para interpretá-las e de autorregulação para sedimentar essas mudanças limitaram o alcance das estratégias. Foi possível observar que abordagens lúdicas como aquelas realizadas com etiquetas nas cores do semáforo e/ou símbolos autoexplicativos, que enunciavam mais a qualidade global do alimento, conquistaram resultados mais definitivos ${ }^{(34,36,37)}$.

Essa característica também foi observada nos estudos que utilizaram transmissão objetiva de informação como estratégia principal de intervenção, através de jornadas, oficinas, seminários, cartazes e panfletos ${ }^{(33,38-42)}$, uma vez que ter o conhecimento sobre a necessidade de consumo de alimentos mais saudáveis e sobre quais grupos alimentares pertenciam não era suficiente, pois no campus da universidade não havia opções para o consumo mais consciente e/ou esses alimentos não eram financeiramente acessíveis $^{(36)}$.

Os resultados desses estudos confirmam a necessidade da sensibilização e da abrangência das estratégias serem intersetoriais e multifacetadas, incluindo desde a disponibilidade de educação nutricional para o aluno até a política e gestão realizadas nas universidades no que diz respeito à disponibilidade de alimentos ${ }^{(26,36)}$. Conclusão essa que pode ser corroborada através do histórico de construção da Política de Segurança Alimentar e Nutricional discutida à luz da intersetorialidade no governo, que traz o desafio e a centralidade dos distintos mecanismos, instrumentos e processos institucionais para a confluência e promoção dessas ações ${ }^{(26,39)}$.

No que tange às ferramentas utilizadas, a maioria dos estudos analisados usaram a internet (através de computadores e/ou dispositivos mobiles) como artifício principal ou auxiliar para envolver os alunos nas ações ${ }^{(31,39,43-48)}$. Esse padrão de estratégia se tornou interessante e alcançou resultados positivos, uma vez que a população universitária, compreendida em sua maioria por jovens adultos entre 18 e 24 anos, possui grande afinidade com tecnologias, bem como, declara ser positivo poder acessar o conteúdo disponibilizado e ter interatividade em qualquer local e horário, sem a necessidade de adaptação de rotina para isso ${ }^{(44)}$.

No caso dos cursos de longa duração ${ }^{(49)}$, as atividades via telefone com feedback simultâneo de aconselhamento ${ }^{(47)}$ e as estratégias com atividades práticas ${ }^{(39)}$, como as oficinas de culinária, embora tenham apresentado resultados positivos, tiveram um alcance do ponto de vista quantitativo limitado. Isso porque essas estratégias exigem maiores recursos financeiros, humanos e de infraestrutura, o que limita sua ação quando o objeto se torna o ensino superior, mesmo sendo bastante relevante na educação básica, para o mesmo fim ${ }^{(20,21,25,50)}$. 
Faz-se necessário discutir, para além das ferramentas e recursos, a abordagem de ensino utilizada nas estratégias, muitas apenas com transferência de informação e com falta de um constructo teórico bem estabelecido ${ }^{(33,34,36,38-42,45)}$. Outras, mais efetivas, com condução baseada na teoria social cognitiva $(\mathrm{TSC})^{(35,46-49)}$, reforçaram o desenvolvimento de autoeficácia e autorregulação por parte dos participantes e, por fim, aquelas com intuito motivacional, também com resultados promissores, sobretudo quando associadas a outras abordagens $\mathrm{s}^{(31,35,43,44,47,51)}$.

Em estudo comparando a abordagem baseada no desenvolvimento de autorregulação, através dos constructos da TSC e uma abordagem motivacional, a primeira se mostrou mais efetiva em mudança de comportamento alimentar ${ }^{(35)}$. No entanto, em outro estudo de grande abrangência, quando comparada uma estratégia motivacional com uma estratégia mais determinada a transmitir quantitativamente o conteúdo, a primeira obteve resultado melhor relacionado à redução do consumo calórico dos participantes, porém inferior à perda de peso, concluindo que ambas seriam importantes se utilizadas juntas ${ }^{(47)}$. O que permite dizer que um somatório dessas abordagens pode ser interessante para estratégias amplas e perenes de educação nutricional.

Os estudos não apresentam, em sua maioria, os responsáveis por realizar as ações de promoção de saúde. Em alguns, sabese que foram aplicadas pelo nutricionista responsável pelo restaurante universitário ${ }^{(52)}$. Em outros, a equipe de gestão em saúde da universidade o fez com objetivo de implementar as Universidades Promotoras de Saúde como estratégias de gestão(39), ou através de parceria com o departamento esportivo da universidade ${ }^{(53)}$. No entanto, a maior parte delas foi desenvolvida por grupos de estudos e pesquisas, através de profissionais da saúde (nutricionistas, psicólogos e educadores físicos) e estudantes de graduação e pós-graduação.

Esse fato reforça uma observação importante relacionada aos trabalhos incluídos na revisão: muitas das estratégias são intervenções avaliativas, objetos de pesquisa, ainda não implementadas nas instituições de maneira definitiva, como parte dos programas institucionais. Em decorrência disso, ressalta-se como limitação do presente estudo a falta de avaliação em longo prazo dos resultados das estratégias e de sua manutenção em benefício da comunidade acadêmica, bem como a ausência de trabalhos realizados no Brasil.

Foi possível, no entanto, concluir que se mostraram menos efetivas as estratégias que se detiveram a apenas transmitir informações aos participantes quando comparadas àquelas que se propuseram a desenvolver autonomia e mecanismos para autorregulação e autogestão em saúde.

Com base no exposto, faz-se necessário um olhar para o desenvolvimento de estratégias de EAN que contemplem as múltiplas facetas de aprendizagem, que sejam efetivas e integradas, ampliando a discussão para os aspectos que permeiam não somente a aquisição de informações, mas sim os determinantes do estilo de vida dos sujeitos e da sociedade em que estão inseridos. Isso permitirá o desenvolvimento da autonomia para uma prática de escolhas alimentares mais saudáveis e perenes, e sua inserção na identidade cultural do ensino superior.

\section{CONCLUSÃO}

O ambiente universitário possui grande potencial para promoção de saúde nutricional e as estratégias mais efetivas foram as que apresentaram uma condução baseada na teoria social cognitiva. No entanto, esse ambiente tem sido pouco explorado em nível nacional e, para mudar essa realidade, faz-se necessária uma ressignificação das estratégias utilizadas e das políticas públicas e ações intersetoriais que sustentem essa prática, inserido-a na identidade social do ensino superior no Brasil.

\section{REFERÊNCIAS}

1. World Health Organization. WHO guidelines approved by the Guidelines Review Committee. Geneva: WHO; 2013.

2. Oliveira NRCD, Paulo UFDS, Padovani RDC et al. Saúde do estudante universitário: uma questão para reflexão. Ciênc Saúde Coletiva. 2014;19(3):995-6.

3. Vasconcelos MM, Coelho PC, Grande IKA. Estado nutricional, consumo alimentar e risco cardiovascular: um estudo em universitários. Nutritional status, food consumption and cardiovascular risk: a study on university students. Rev Nutr. 2009;22(6):837-46.

4. Neville MM, Geppert J, Min Y, Grimble G, Crawford MA, Ghebremeskel K. Dietary fat intake, body composition and blood lipids of university men and women. Nutr Health. 2012;21(3):173-85.

5. Greene GW, Schembre SM, White AA, Hoerr SL, Lohse B, Shoff S, et al. Identifying clusters of college students at elevated health risk based on eating and exercise behaviors and psychosocial determinants of body weight. J Am Diet Assoc. 2011;111(3):394-400.

6. Cooke R, Papadaki A. Nutrition label use mediates the positive relationship between nutrition knowledge and attitudes towards healthy eating with dietary quality among university students in the UK. Appetite. 2014;83:297-303. 
7. Tavolacci MP, Grigioni S, Richard L, Meyrignac G, Dechelotte P, Ladner J. Eating disorders and associated health risks among university students. J Nutr Educ Behav. 2015;47(5):412-20.

8. Chourdakis M, Tzellos T, Papazisis G, Toulis K, Kouvelas D. Eating habits, health attitudes and obesity indices among medical students in northern Greece. Appetite. 2010;55(3):722-5.

9. El Ansari W, Stock C, Snelgrove S, Hu X, Parke S, Davies S, et al. Feeling healthy? A survey of physical and psychological wellbeing of students from seven universities in the UK. Int J Environ Res Public Health. 2011;8(5):1308-23.

10. Batista M Filho, Rissin A. A transição nutricional no Brasil: tendências regionais e temporais. Cad Saúde Pública. 2003;19(Supl 1):181-91.

11. Souza EBD. Transição nutricional no Brasil: análise dos principais fatores. Cad UniFOA. 2010;5(13):49-53.

12. Ministério da Saúde (BR). Uma análise da situação de saúde e das doenças transmissíveis relacionadas à pobreza. Brasília: Ministério da Saúde; 2014.

13. Ministério da Saúde (BR). Vigitel Brasil 2010: vigilância de fatores de risco e proteção para doenças crônicas por inquérito telefônico. Brasília: Ministério da Saúde; 2011.

14. Ministério da Saúde (BR). PNAN Política Nacional de Alimentação e Nutrição. Brasília: Ministério da Saúde; 2013.

15. Albaneide MLP. A trajetória do Programa Nacional de Alimentação Escolar no período de 2003-2010: relato do gestor nacional. Ciênc Saúde Coletiva. 2013;18(4):909-16.

16. Guedes FAV, Garcia CG, Mantovani TCS, Gonçalves LC. Reflexões sobre a atuação do nutricionista no Programa Nacional de Alimentação Escolar no Brasil. Ciênc Saúde Coletiva. 2013;18(4):917-26.

17. Gomes JP. As escolas promotoras de saúde: uma via para promover a saúde e a educação para a saúde da comunidade escolar. Educação. 2009;32(1):84-91.

18. Cardoso V, Ielusc J, Reis APD, Iervolino SA. Escolas promotoras de saúde. Rev Bras Crescimento Desenvolv Hum. 2008;18(2):107-15.

19. Loureiro I. A importância da educação alimentar: o papel das escolas promotoras de saúde. Rev Port Saúde Pública. 2004;22(2):43-55.

20. Rodrigues EM, Lima JFJ, Santos JP, Carvalho AE, Chagas CG, Fernandes MMN. Validação de metodologias ativas de ensino- aprendizagem na promoção da saúde alimentar infantil. Rev Nutr. 2012;25(1):79-88.

21. Casemiro JP. Impasses, challenges, and interfaces of food and nutrition education as a process of popular participation. Trab Educ Saúde. 2015;13(2):493-514.

22. Camozzi ABQ, Menezes IHCF, Silva PO. Promoção da Alimentação Saudável na Escola: realidade ou utopia? Cad Saúde Colet (Rio J). 2015;23(1):32-7.

23. Figueiredo TAM, Abreu MMSA. A saúde na escola: um breve resgate histórico. Ciênc Saúde Coletiva. 2010;15(2):397402.

24. Ministério da Saúde (BR). Diretrizes metodológicas: elaboração de revisão sistemática e metanálise de ensaios clínicos randomizados. Brasília: Ministério da Saúde; 2012. (Série A. Normas e Manuais Técnicos).

25. Carvalho RT, Yokota TFDV, Pinheiro ARO, Schmitz BAS, Coitinho DC, Rodrigues MLCF. Projeto "a escola promovendo hábitos alimentares saudáveis”: comparação de duas estratégias de educação nutricional no Distrito Federal, Brasil. Rev Nutr. 2010;23(1):37-47.

26. Burlandy L. A construção da política de segurança alimentar e nutricional no Brasil: estratégias e desafios para a promoção da intersetorialidade no âmbito federal de governo.(Report). Ciênc Saúde Coletiva. 2009;14(3):851-60.

27. Rodríguez RF, Palma X, Romo A, Escobar D, Aragú GB, Espinoza OL, et al. Hábitos alimentarios, actividad física y nivel socioeconómico en estudiantes universitarios de Chile. Nutrición Hospitalarial. 2013;28(2):447-55.

28. Fontes ACD, Vianna RPT. Prevalência e fatores associados ao baixo nível de atividade física entre estudantes universitários de uma universidade pública da região Nordeste - Brasil. Rev Bras Epidemiol. 2009;12(1):20-9.

29. Simão AF, Precoma DB, Andrade JP, Correa H Filho, Saraiva JFK, Oliveira GMM, et al. I Diretriz Brasileira de Prevenção Cardiovascular. Arq Bras Cardiol. 2013;101(6 Supl 2):1-63. 
30. Diretrizes da sociedade Brasileira de Diabetes 2014-2015. São Paulo: AC Farmacêutica; 2015.

31. Shahril MR. A 10-Week Multimodal Nutrition Education Intervention Improves Dietary Intake among University Students: Cluster Randomized Controlled Trial. J Nutrition Metabolism. 2013;2013:1-11.

32. Ministério da Saúde (BR). Plano de Ações Estratégicas para o enfrentamento das Doenças Crônicas não Transmissíveis (DCNT) no Brasil 2011-2022. Brasília: Ministério da Saúde; 2011.

33. Jimenéz MJ, Margalef MI, Casermeiro MA, Gasparini SR, Guanca R, Martinez Bustos M, et al. Evaluación de intervenciones educativas en el conocimiento y opinión de la comunidad universitaria sobre alimentos funcionales. Evaluation of educational interventions in the university community's knowledge and opinion on functional foods. Diaeta. 2010;28(132):23-8.

34. Abbot JM. Development and evaluation of a university campus-based food safety media campaign for young adults. J Food Prot. 2012;75(6):1117-24.

35. Lhakhang P. A brief intervention increases fruit and vegetable intake. A comparison of two intervention sequences. Appetite. 2014;82:103-10.

36. Feldman C. Using student opinion and design inputs to develop an informed university foodservice menu. Appetite. 2013;69:80-8.

37. Hieke S, Wilczynski P. Colour Me In--an empirical study on consumer responses to the traffic light signposting system in nutrition labelling. Public Health Nutr. 2012;15(5):773-82.

38. Sánchez SV, Fornons FD, Aguilar MA, Vaqué CC, Villarroel MR. Evolución de los conocimientos acerca de los trastornos del comportamiento alimentario, através de una intervención educativa en estudiantes de enseñanza post- obligatoria. Nutr Clín Diet Hosp. 2015;35(1):32-41.

39. Becerra-Bulla F, Pinzón-Villate G, Vargas-Zárate M. Building a health promotion programme based in diet and nutrition at the Universidad Nacional de Colombia, Bogotá hacia la creación del programa universidad promotora de la salud desde la alimentación y nutrición en la universidad nacional de Colombia, sede Bogotá. Rev Fac Med (Bogotá). 2011;59(Supl 1):67-76.

40. Anetor GO, Ogundele BO, Oyewole OE. Effect of nutrition education on the eating habits of undergraduates in SouthWest, Nigeria. Asian J Epidemiology. 2012;5(2):32-41.

41. Higgins SJW, Lauzon LL, Yew AC, Bratseth CD, McLeod N, Marks R. Wellness 101: health education for the university student. Asian J Epidemiology. 2010;110(4):309-327.

42. Vijayapushpam T, Antony GM, Rao GM, Rao DR. Nutrition and health education intervention for student volunteers: topic-wise assessment of impact using a non-parametric test. Public Health Nutr. 2009;13(1):131-6.

43. ODonnell S, Greene GW, Blissmer B. The Effect of Goal Setting on Fruit and Vegetable Consumption and Physical Activity Level in a Web-Based Intervention. J Nutr Educ Behav. 2014;46(6):570-5.

44. Dour CA, Horacek TM, Schembre SM, Lohse B, Hoerr S, Kattelmann K, et al. Process evaluation of Project WebHealth: a nondieting Web-based intervention for obesity prevention in college students. J Nutr Educ Behav. 2013;45(4):288-95.

45. Harvey-Berino J, Pope L, Gold BC, Leonard H, Belliveau C. Undergrad and overweight: an online behavioral weight management program for college students. J Nutr Educ Behav. 2012;44(6):604-8.

46. Poddar KH. Dairy intake and related self-regulation improved in college students using online nutrition education. J Acad Nutr Diet. 2012;112(12):1976-86.

47. Pearson ES, Irwin JD, Morrow D, Battram DS, Melling CW. The CHANGE Program: comparing an interactive vs. prescriptive approach to self-management among university students with obesity. Can J Diabetes. 2013;37(1):4-11.

48. Poddar KH, Hosig KW, Anderson ES, Nickols-Richardson SM, Duncan SE. Web-based nutrition education intervention improves self-efficacy and self-regulation related to increased dairy intake in college students. J Am Diet Assoc. 2010;110(11):1723-7.

49. Ha EJ, Caine-Bish N. Interactive introductory nutrition course focusing on disease prevention increased whole-grain consumption by college students. J Nutr Educ Behav. 2011;43(4):263-7.

50. Silva LAS, Braga JP, Lima AM, Vieira GAF, Ramos LS, Soares MCF. O nutricionista no programa de alimentação escolar: avaliação de uma experiência de formação a partir de grupos focais. The dietician in the school food program: evaluation of a training experience based on focus groups. Rev Nutr. 2012;25(1):107-17. 
51. Ungar N, Sieverding M, Stadnitski T. Increasing fruit and vegetable intake. "Five a day" versus "just one more". Appetite. 2013;65:200-4.

52. Pohlmeier A, Reed DB, Boylan M, Harpet S. Using focus groups to develop a nutrition labeling program within university food service. Fam Consum Sci Res J. 2015;40(4):431-43.

53. Karpinski C. Exploring the feasibility of an academic course that provides nutrition education to collegiate student-athletes. J Nutr Educ Behav. 2012;44(3):267-70.

\section{Endereço do primeiro autor:}

Marina Carvalho Berbigier

Rua Sarmento Leite, 245

Bairro: Centro

CEP: 90050-170 - Porto Alegre - RS - Brasil

E-mail: marina.berbigier@gmail.com 